\title{
Comparative analysis of circular RNAs between soybean cytoplasmic male-sterile line NJCMS1A and its maintainer NJCMS1B by high-throughput sequencing
}

Linfeng Chen ${ }^{1}$, Xianlong Ding ${ }^{1}$, Hao Zhang ${ }^{1}$, Tingting He${ }^{1}$, Yanwei $\mathrm{Li}^{1}$, Tanliu Wang ${ }^{1}$, Xiaoqiang $\mathrm{Li}^{1}$, Ling Jin ${ }^{1}$, Qijian Song ${ }^{2}$, Shouping Yang ${ }^{1 *}$ and Junyi Gai ${ }^{1^{*}}$

\begin{abstract}
Background: Cytoplasmic male sterility (CMS) is a natural phenomenon of pollen abortion caused by the interaction between cytoplasmic genes and nuclear genes. CMS is a simple and effective pollination control system, and plays an important role in crop heterosis utilization. Circular RNAs (circRNAs) are a vital type of non-coding RNAs, which play crucial roles in microRNAs (miRNAs) function and post-transcription control. To explore the expression profile and possible functions of circRNAs in the soybean CMS line NJCMS1A and its maintainer NJCMS1B, high-throughput deep sequencing coupled with RNase R enrichment strategy was conducted.

Results: CircRNA libraries were constructed from flower buds of NJCMS1A and its maintainer NJCMS1B with three biological replicates. A total of 2867 circRNAs were identified, with 1009 circRNAs differentially expressed between NJCMS1A and NJCMS1B based on analysis of high-throughput sequencing. Of the 12 randomly selected circRNAs with different expression levels, 10 showed consistent expression patterns based on high-throughput sequencing and quantitative real-time PCR analyses. Tissue specific expression patterns were also verified with two circRNAs by quantitative real-time PCR. Most parental genes of differentially expressed circRNAs were mainly involved in biological processes such as metabolic process, biological regulation, and reproductive process. Moreover, 83 miRNAs were predicted from the differentially expressed circRNAs, some of which were strongly related to pollen development and male fertility; The functions of miRNA targets were analyzed using Gene Ontology (GO) and Kyoto Encyclopedia of Genes and Genomes (KEGG), and the target mRNAs were significantly enriched in signal transduction and programmed cell death. Furthermore, a total of 165 soybean circRNAs were predicted to contain at least one internal ribosome entry site (IRES) element and an open reading frame, indicating their potential to encode polypeptides or proteins.
\end{abstract}

Conclusions: Our study indicated that the circRNAs might participate in the regulation of flower and pollen development, which could provide a new insight into the molecular mechanisms of CMS in soybean.

Keywords: Soybean (Glycine max (L.) Merr.), Cytoplasmic male sterility, CircRNAs, High-throughput sequencing, Parental genes, Binding miRNAs

\footnotetext{
*Correspondence: spyung@126.com; sri@njau.edu.cn

${ }^{1}$ Soybean Research Institute, National Center for Soybean Improvement, Key Laboratory of Biology and Genetic Improvement of Soybean (General,

Ministry of Agriculture), State Key Laboratory of Crop Genetics and

Germplasm Enhancement, Jiangsu Collaborative Innovation Center for

Modern Crop Production, Nanjing Agricultural University, Nanjing 210095,

China

Full list of author information is available at the end of the article
}

(c) The Author(s). 2018 Open Access This article is distributed under the terms of the Creative Commons Attribution 4.0 International License (http://creativecommons.org/licenses/by/4.0/), which permits unrestricted use, distribution, and reproduction in any medium, provided you give appropriate credit to the original author(s) and the source, provide a link to the Creative Commons license, and indicate if changes were made. The Creative Commons Public Domain Dedication waiver (http://creativecommons.org/publicdomain/zero/1.0/) applies to the data made available in this article, unless otherwise stated. 


\section{Background}

Soybean (Glycine $\max (\mathrm{L}$.) Merr.) is an important oil and protein crop, but soybean yield is low. Utilization of heterosis has been proved to be one of the effective methods to increase crop yield, and cytoplasmic male sterility (CMS) plays an important role in heterosis utilization [1]. CMS is a maternally inherited phenotype caused by the interaction of cytoplasmic genes and nuclear genes, which lead to pollen abortion but normal pistil development [2,3]. To date, CMS has been observed in over 200 plant species [4] and is often obtained in wild germplasm or by inter- or intra-subspecies backcrossing [5]. In soybean, CMS was first reported by Davis [6], after which many studies on CMS were conducted [7, 8]. In recent years, transcriptomics [9], proteomics [10], microRNA [11], and DNA methylation of CMS have been explored [12], but the function of circular RNAs (circRNAs) in soybean CMS has not been reported.

CircRNAs are a class of endogenous noncoding RNAs, which do not have $5^{\prime}$ caps and $3^{\prime}$ tails and form a ring structure with covalent bonds [13]. CircRNAs were first reported based on deep sequencing of RNA by Salzman et al. [14], which were neglected for decades because they were considered as transcriptional noise or reverse transcription PCR artifacts by-product [15]. With the development of high-throughput sequencing technology and bioinformatics, circRNAs have been identified in all domains of life, including eukaryotes [15, 16], archaea [17], bacteria [18], and viruses [19]. Previous studies showed that circRNAs were more resistant to degradation by RNase $\mathrm{R}$ than their linear counterparts [20], and had tissue, cell-type, and developmental-stage specific expression patterns [15, 21, 22]. Li et al. [23] demonstrated that the circRNAs located in the nucleus could promote transcription of their parental genes via specific RNA-RNA interactions, but another recent study revealed that circRNAs derived from organelle genome could also regulate gene expression [24]. Furthermore, circRNAs act as miRNA sponges and prevent them from inhibiting their target mRNAs $[25,26]$. Most recent studies have demonstrated that some circRNAs can be translated into polypeptides or proteins by translation initiation element internal ribosome entry site (IRES) or N6-methyladenosine $\left(\mathrm{m}^{6} \mathrm{~A}\right)[27,28]$.

Although not as comprehensive as in animals, the exploration of circRNAs in plants is increasing. Differential expression of circRNAs has been reported in plants, for example, 27 rice exonic circRNAs were associated with phosphate starvation responsive expression [16], 163 tomato circRNAs with chilling responsive expression [29], and 62 wheat circRNAs with dehydration stress specific expression [30]. Moreover, some circRNAs showed time, tissue, species, or developmental-stage specific expression patterns in plants [16, 24, 31, 32]. Unlike the positive regulation of circRNAs in animals [23], over-expression of circRNAs in rice and tomato reduced expression level of their parental genes [33, 34]. In soybean, a total of 5372 circRNAs were identified, of which approximately $80 \%$ were generated from paralogous genes [35]. Meanwhile, Zhao et al. [35] found that up-regulation of circRNAs might decrease the activity of target miRNAs and increase expression of the related mRNAs.

To explore the expression profile and possible functions of circRNAs in the soybean CMS line NJCMS1A vs. its maintainer line NJCMS1B, high-throughput deep sequencing coupled with RNase $R$ enrichment strategy was conducted. Target miRNAs of differentially expressed circRNAs and the correlated mRNAs were predicted using bioinformatics methods, and their potential functions were further analyzed. Our study investigated the possible role of circRNAs in CMS for the first time, and the results showed that the circRNAs might participate in the regulation of flower and pollen development in soybean.

\section{Methods}

\section{Plant materials and sample collection}

The soybean cytoplasmic male-sterile line NJCMS1A was developed through consecutive backcross. The cultivar N8855 was the donor and cultivar N2899 (designated as NJCMS1B afterwards) was a recurrent parent [8, 36, 37]. NJCMS1A and NJCMS1B are near-isogenic lines of isonuclear alloplasmic type with similar nucleus but different cytoplasm. NJCMS1A and NJCMS1B were grown in the summer of 2016 at Dangtu Experimental Station, National Center for Soybean Improvement, Nanjing Agricultural University, Maanshan, Anhui, China. Male sterile plants were identified by observing dehiscence of anthers, germination rate of pollen, and morphological traits of plants at maturity. Because it is difficult to determine development stage of pollen based on flower bud size in soybean, during the flowering period, the flower buds of different sizes were collected from NJCMS1A or NJCMS1B plants and mixed, with three biological replicates per line. Meanwhile, roots, stems and leaves of soybean were also collected at the flowering period with three biological replicates. The tissues were immediately frozen in liquid nitrogen and stored at $-80{ }^{\circ} \mathrm{C}$ for further use.

\section{Total RNA extraction and RNase $\mathrm{R}$ treatment}

Total RNA from flower buds of NJCMS1A and NJCMS1B lines was extracted using the RNAprep pure Plant Kit (Qiagen, DEU) according to the manufacturer's protocol, DNA contamination was removed using the DNase I contained in the Kit. For RNase R-treated total 
RNA samples, the purified DNasel-treated total RNA was incubated for $15 \mathrm{~min}$ at $37{ }^{\circ} \mathrm{C}$ with 3 units RNase $\mathrm{R}$ per $\mu \mathrm{g}$ of total RNA (Epicentre, Shanghai, CN). RNA was subsequently purified using an RNase MinElute Cleaning Kit (Qiagen, DEU). To obtain accurate and sufficient transcriptome data, RNA from three biological replicates of NJCMS1A and NJCMS1B lines were sequenced.

\section{CircRNA library construction and sequencing}

rRNA-depleted RNAs in $5 \mu \mathrm{g}$ RNA per sample was obtained using the Epicentre Ribo-zero ${ }^{\mathrm{rm}}$ rRNA Removal Kit (Epicentre, USA) and were further treated with RNase R (Epicentre, USA) for Trizol extraction. Sequencing libraries were prepared using NEBNext Ultra Directional RNA LibraryPrep Kit (NEB, USA) following manufacturer's instructions. The libraries were preliminarily quantified by Qubit and diluted to $1 \mathrm{ng} / \mathrm{ul}$. The insert size of the libraries was detected by Agilent 2100/ Caliper, which was expected to be distributed around $250 \sim 300 \mathrm{bp}$. The effective concentration of the libraries was accurately quantified by qRT-PCR, and the effective concentration was greater than $2 \mathrm{nM}$ to ensure the libraries quality. Briefly, fragmentation was carried out using divalent cations under elevated temperature in NEBNext First-strand Synthesis Reaction Buffer. First-strand cDNA was synthesized using random hexamer primer and M-MuLV reverse transcriptase (RNaseH). Second-strand cDNA synthesis was subsequently performed using DNA Polymerase I and RNase $H$. In the reaction buffer, dTTP in dNTPs was replaced by dUTP. Remaining overhangs were blunted via exonuclease/polymerase. After adenylation of 3' ends of DNA fragments, NEBNext Adaptor with hairpin loop structure was ligated for hybridization. In order to obtain cDNA fragments of 150-200 bp in length, the library fragments were purified with the AMPure XP system (Beckman Coulter, Beverly, USA). Subsequently, $3 \mu$ USER Enzyme (NEB, USA) was added to adaptor-ligated cDNA of $150-200 \mathrm{bp}$ and kept at $37{ }^{\circ} \mathrm{C}$ for $15 \mathrm{~min}$ followed by $5 \mathrm{~min}$ at $95{ }^{\circ} \mathrm{C}$ before PCR. PCR was performed with Phusion high-fidelity DNA polymerase, universal PCR primer, and index (X) primer. Finally, the library was purified (AMPure XP system) and then qualified by the Agilent Bioanalyzer 2100 system. Clustering of the index-coded samples was performed on a cBot Cluster Generation System using HiSeq PE Cluster Kit v4 cBot (Illumina) according to the manufacturer's instructions. After cluster generation, the libraries were sequenced on an Illumina Hiseq 2500 platform and 125 bp paired-end reads were generated. Library construction and sequencing were carried out by Novogene (Novogene, Beijing, China).

\section{Identification of circRNAs and differential expression analysis}

Raw data (raw reads in fastq format) was first processed by a custom perl script. Sequence reads with adapter, ploy-N, and low-quality were eliminated. The remaining reads were used for read quality and GC content estimation, and downstream calculation. Soybean reference genome and gene annotation were downloaded from the website at https://phytozome.jgi.doe.gov/pz/portal.html\#. The index of the reference genome was built using Bowtie v2.0.6, paired-end reads were aligned to the reference genome (soybean genome version 2.0 in Phytozome) using TopHat v2.0.9. 20-mers from $5^{\prime}$ and $3^{\prime}$ end of the unmapped reads were extracted and aligned independently to reference sequences using Bowtie v2.0.6. Anchor sequences were extended by find_circ [15], such that the complete read was aligned and the breakpoints were flanked by GU/AG splice sites. Subsequently, the back-spliced reads with at least two supporting reads were annotated as circRNAs.

Expression level of circRNAs was normalized by transcript per million (TPM) through the following criteria [38]: Normalized expression $=($ mapped $\mathrm{reads}) /($ total reads) * 1000000. Differential expression between two groups was performed using DESeq2 (version 1.6.3) [39]. Differentially expressed circRNAs were identified with the cutoff threshold of $\mid \log _{2}$ (fold-changes) $\mid \geq 2$ based on the method used by Wang et al. [30] and Liu et al. [31].

\section{Prediction of miRNA targets of circRNAs, mRNA targets of miRNA, and annotation of functions}

miRNA binding sites of differentially expressed circRNAs were predicted by psRobot_tar in psRobot [40]. Meanwhile, the circRNA-miRNA interaction network was delineated by Cytoscape [41]. The obtained miRNAs were used to predict the target mRNAs by psRobot with default parameters [40]. The parental genes of differentially expressed circRNAs and the predicted target mRNAs were classified into different functional processes based on Gene Ontology (GO) term enrichment using the Web Gene Ontology Annotation Plot (WEGO) [42] and agriGO [43, 44], respectively. The KOBAS [45] was used for KEGG pathways enrichment analysis. The gene annotation of $A$. thaliana at http://www.arabidopsis.org/ was used to define functions of homologous genes in soybean.

\section{PCR amplification and sanger sequencing}

To validate the soybean circRNAs identified in this study, a set of 12 differentially expressed circRNAs were amplified with divergent primer. As a control, a pair of convergent primers were designed for gma-circRNA0002 (Additional file 1). All primers were synthesized by Invitrogen (Shanghai, China). The total and RNase R-treated 
RNA of each sample were used as templates. PCR products were separated using agarose gel, and each band was excised and purified using the AxyPrep DNA Gel Extraction Kit (Axygen, Suzhou, China) for Sanger-sequencing.

\section{Quantitative real-time PCR validation}

Quantitative real-time PCR (qRT-PCR) was carried out to validate differential expressional levels of circRNAs. Divergent primers were designed in order to obtain amplicon from circle template (Additional file 1). According to the instruction of the iScript Select cDNA Synthesis Kit (BIO-RAD, USA), $1 \mu \mathrm{g}$ of the total RNA untreated with RNase $R$ was reverse-transcribed with random primers. Expression of circRNAs was quantified using the iTaq Universal SYBR Green Supermix (BIO-RAD, USA) on the Bio-Rad CFX96 machine (CFX96 Touch, BIO-RAD, USA). All real-time PCR assays were performed with three biological replicates, and the expression of the housekeeping gene GADPH was used as a reference for data normalization. The qRT-PCR aliquot contained $2 \mu \mathrm{L}$ cDNA, $0.6 \mu \mathrm{L}$ of each upstream and downstream primers $(10 \mu \mathrm{M}), 10 \mu \mathrm{L}$ Takara SYBR Premix Ex Taq, and $6.8 \mu \mathrm{L}$ RNase-free $\mathrm{ddH}_{2} \mathrm{O}$ and performed with an initial denaturation at $95{ }^{\circ} \mathrm{C}$ for $30 \mathrm{~s}$, followed by 40 cycles at $95{ }^{\circ} \mathrm{C}$ for $5 \mathrm{~s}, 60{ }^{\circ} \mathrm{C}$ for $30 \mathrm{~s}$. The amplification curve and melting curve were examined to evaluate specific amplification. The circRNAs relative expression levels ( $\log _{2}$ values) were calculated using the $2^{-\Delta \Delta \mathrm{Ct}}$ method. Student's t-test was performed to compare differences of circRNAs expression between NJCMS1A and NJCMS1B. The probability threshold of significance was $P<0.05$.

\section{Protein-coding potential prediction of circRNAs}

The IRES element is required to initiate translation of a mRNA sequence without a $5^{\prime}$-cap structure [46]. If a circRNA has at least one IRES element, it may be able to encode a protein. To predict the IRES elements in soybean circRNAs, we blasted sequences of the circRNAs to all the IRES sequences in the website (http://iresite.org/) at an E-value < 0.05 [47]. To predict the ORFs of predictive circRNAs, we used the prediction algorithm at the website (https://github.com/kadenerlab/cORF_pipeline). Briefly, the longest ORF spanning the circRNA junction was selected for further analysis. The possible coding products of the circRNAs with protein-coding potential were used to predict the conserved domains using the Conserved Domain Database (https://www.ncbi.nlm.nih.gov/ Structure/cdd/wrpsb.cgi).

\section{Results and discussion}

\section{Identification of circRNAs in soybean}

Total amount of sequence for each RNA libraries was $\geq 9$ Gb with Q20 $\geq 93 \%$, Q30 $\geq 85 \%$, and an error rate $\leq 0.05$ (Table 1), the depth and accuracy of high quality sequence was sufficient for subsequent analysis.

A total of 2867 circRNAs were identified, which included 1722 from NJCMS1B and 1643 from NJCMS1A (Fig. 1a, Additional file 2). Among them, 452 (15.8\%) were derived from exons of a single protein-coding gene (exonic circRNAs), 821 (28.6\%) from introns (intronic circRNAs), 293 (10.2\%) from exons and introns of one or more genes (exon-intron circRNAs), and 1301 (45.4\%) from intergenic regions (intergenic circRNAs) (Fig. 1b). CircRNAs primarily derived from exons in several plants have been reported, e.g. 5152 (85.7\%) circRNAs in A. thaliana [16], 615 (72.0\%) in tomato [29], and $1453(93.7 \%)$ in maize [48]. However, only 2494 (46.4\%) exonic circRNAs were identified in soybean by Zhao et al. [35], which was similar to our result. The length of circRNAs in this study ranged from 150 to $44,756 \mathrm{bp}$, but most $(92.8 \%)$ were $<2000$ bp (Fig. 1c). The length distribution of soybean circRNAs in this study was similar to that reported by Zhao et al. [35]. Additionally, the sequence alignment between the circRNAs inform this study and previous study (Zhao et al. [35]) using blastn indicated that only 78 circRNAs were homologous (Additional file 3 ) at e-value $<1 \mathrm{e}^{-5}$ and identity $>85 \%$. The small proportion of homologous circRNAs in these two studies might be associated with tissue specific expression pattern of circRNA (Additional file 4) and use of different circRNAs prediction software. Besides, the number of circRNAs in different chromosomes and the densities of circRNAs in different chromosomal regions were also different (Fig. 1d).

\section{Expression profiling of circRNAs and qRT-PCR validation} A total of 1009 circRNAs with at least two-fold $(\log 2)$ change of expression level between NJCMS1A and NJCMS1B were identified by high-throughput sequencing. Of these, a total of 360 circRNAs were up-regulated, 649 circRNAs were down-regulated in NJCMS1A vs. NJCMS1B (Additional file 5).

The back splicing sites and expression profiles of the 12 randomly selected from 1009 circRNAs were further experimentally validated. We treated total RNA samples with RNase $\mathrm{R}$ to verify the special stability (resistance to exonuclease-mediated degradation) of circRNAs and eliminated interference from linear RNA. Divergent primers were designed to guarantee that amplifications were from circular templates and a pair of convergent primers for gma-circRNA0002 were designed as a control. Total RNA samples and RNase R-treated samples were used as reaction templates to amplify circRNAs. As 
Table 1 Summary of circRNA sequencing data

\begin{tabular}{lllllllll}
\hline Sample & Replicates & $\begin{array}{l}\text { Number of } \\
\text { raw reads }\end{array}$ & $\begin{array}{l}\text { Number of } \\
\text { clean reads }\end{array}$ & $\begin{array}{l}\text { Clean bases } \\
(\mathrm{Gb})\end{array}$ & $\begin{array}{l}\text { Error rate } \\
(100 \%)\end{array}$ & $\begin{array}{l}\text { Q20 } \\
(100 \%)\end{array}$ & $\begin{array}{l}\text { Q30 content } \\
(100 \%)\end{array}$ & $\begin{array}{l}\text { GC } \\
(100 \%)\end{array}$ \\
\hline \multirow{3}{*}{ NJCMS1A } & NJCMS1A-1 & $84,474,228$ & $80,699,898$ & 12.1 & 0.05 & 93.38 & 85.41 & 54.16 \\
& NJCMS1A-2 & $67,528,014$ & $63,913,764$ & 9.58 & 0.02 & 97.85 & 94.07 & 53.75 \\
& NJCMS1A-3 & $98,550,080$ & $92,713,788$ & 13.9 & 0.03 & 97.29 & 93.12 & 54.55 \\
\multirow{3}{*}{ NJCMS1B } & NJCMS1B-1 & $74,471,198$ & $68,964,198$ & 10.34 & 0.03 & 97.39 & 93.08 & 53.11 \\
& NJCMS1B-2 & $77,162,554$ & $70,527,006$ & 10.58 & 0.03 & 97.04 & 93.03 & 53.94 \\
& NJCMS1B-3 & $88,067,914$ & $84,489,922$ & 12.68 & 0.05 & 93.53 & 85.55 & 54.67 \\
\hline
\end{tabular}

a result, all 12 pairs of divergent primers yielded amplification products with the expected length from cDNAs that with and without RNase R treatment. To demonstrate that the amplification products from the divergent primers were derived from the corresponding circRNAs and spanning the junction sites, we collected the amplification products for further detection by Sanger sequencing (Fig. 2 and Additional file 6). As shown in Fig. 3, among the 12 random-selected circRNAs, 10 showed consistent expression patterns with RNA-seq results. The coincidence rate between qRT-PCR and RNA-seq was $83.3 \%$. Two inconsistent expression of circRNAs candidates (gma-circ2468 and gma-circ2848) may be caused by the low expression levels of the two circRNAs.
A

NJCMS1B

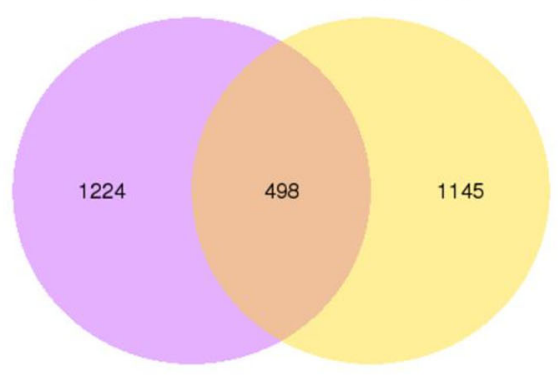

C

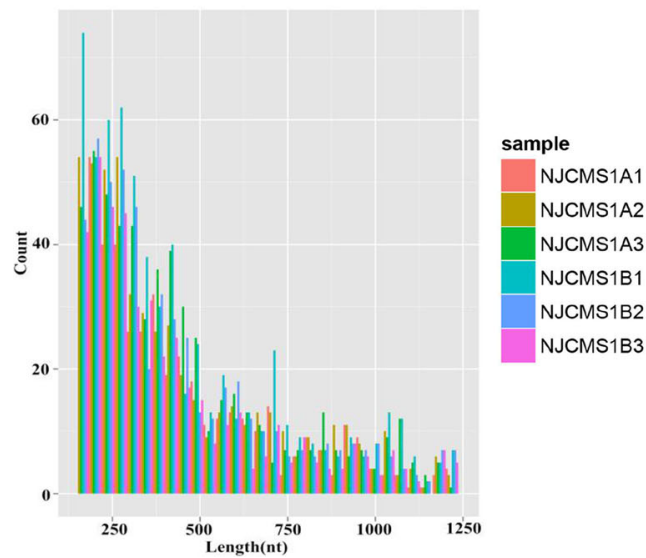

B

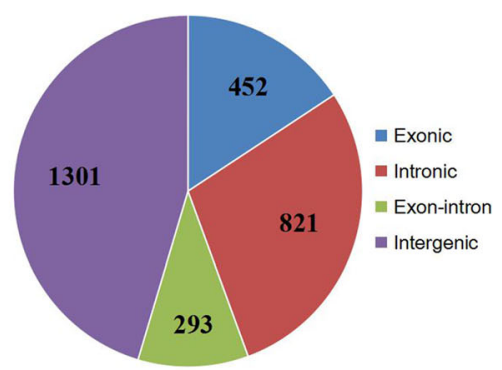

$\mathrm{D}$

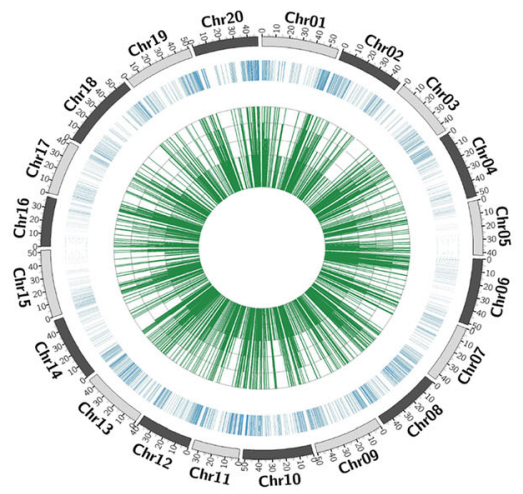

Fig. 1 CircRNA sequencing data. (a) Venn diagram shows the number of the identified circRNAs in NJCMS1A and NJCMS1B. (b) Source statistics of the circRNAs. (c) Sequence length distribution of the circRNAs in different samples. (d) Circle plot shows the distribution of the identified circRNAs in soybean chromosomes and their expression levels. The outermost layer represents all soybean chromosomes (Chr01-Chr20). The middle blue lines show the distribution of the circRNAs in soybean chromosomes, while the denser lines indicate more circRNA distribution. The innermost green lines show expression levels of the circRNAs, and the height of the lines indicates the level of expression 


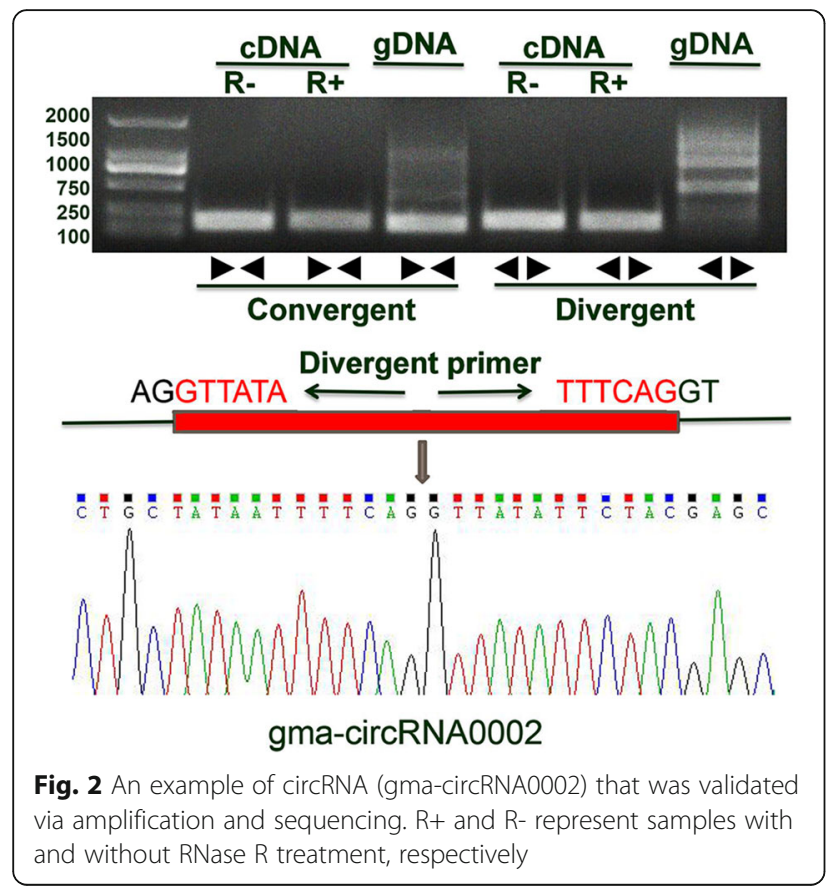

Tissue specific expression patterns validation by qRT-PCR Previous studies showed that circRNAs were tissuepreferentially expressed $[15,24]$. To verify this, two highly expressed circRNAs (gma-circRNA0002 and gma-circRNA2483) based on the high throughput sequencing were selected for qRT-PCR analysis, these two

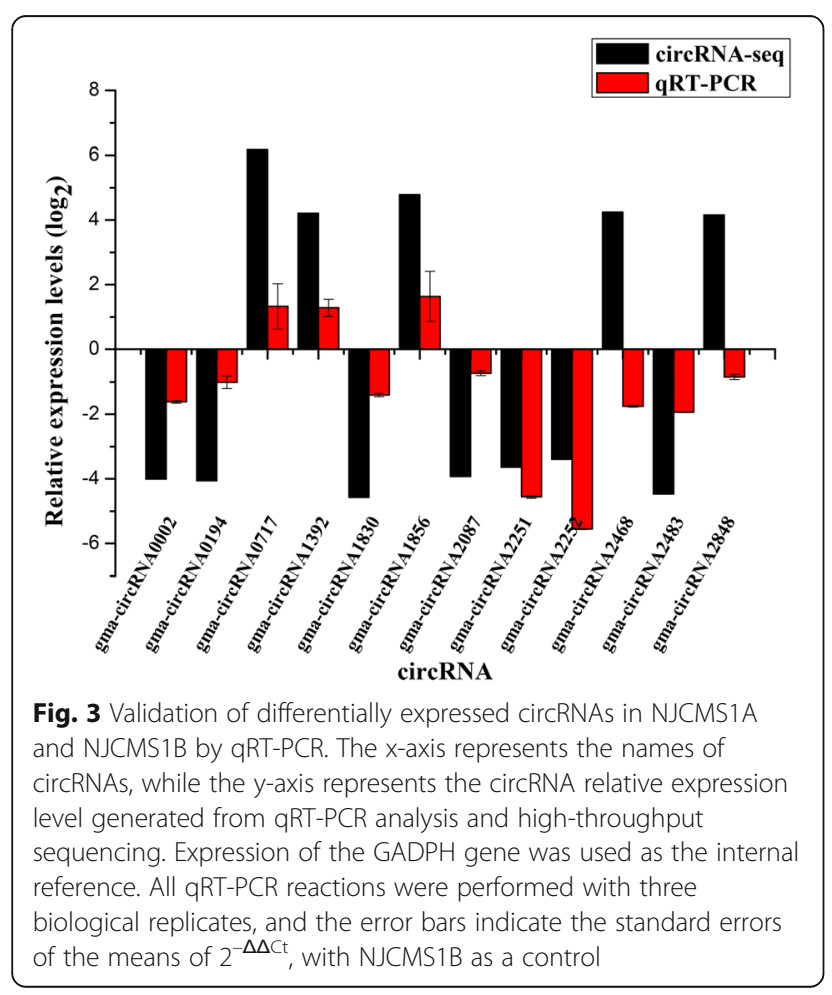

circRNAs were expressed only in leaves and flower buds of NJCMS1A and NJCMS1B, but not in roots and stems (Fig. 4). The expression level of gma-circRNA0002 was only significantly different in the flower buds, while the expression levels of gma-circRNA2483 were significantly different in the leaves and flower buds between NJCMS1A and NJCMS1B.

\section{Functional categorization of parental genes of} differentially expressed circRNAs

It has been reported that circRNAs could regulate expression of their parental genes [33, 34]. To understand the possible functions of circRNAs in CMS of soybean, the parental genes of the differentially expressed circRNAs were predicted. A total of 545 parental genes were obtained from the 1009 differentially expressed circRNAs. GO classification showed that these genes are involved in a wide range of biological processes, such as in metabolic process (GO:0008152), biological regulation

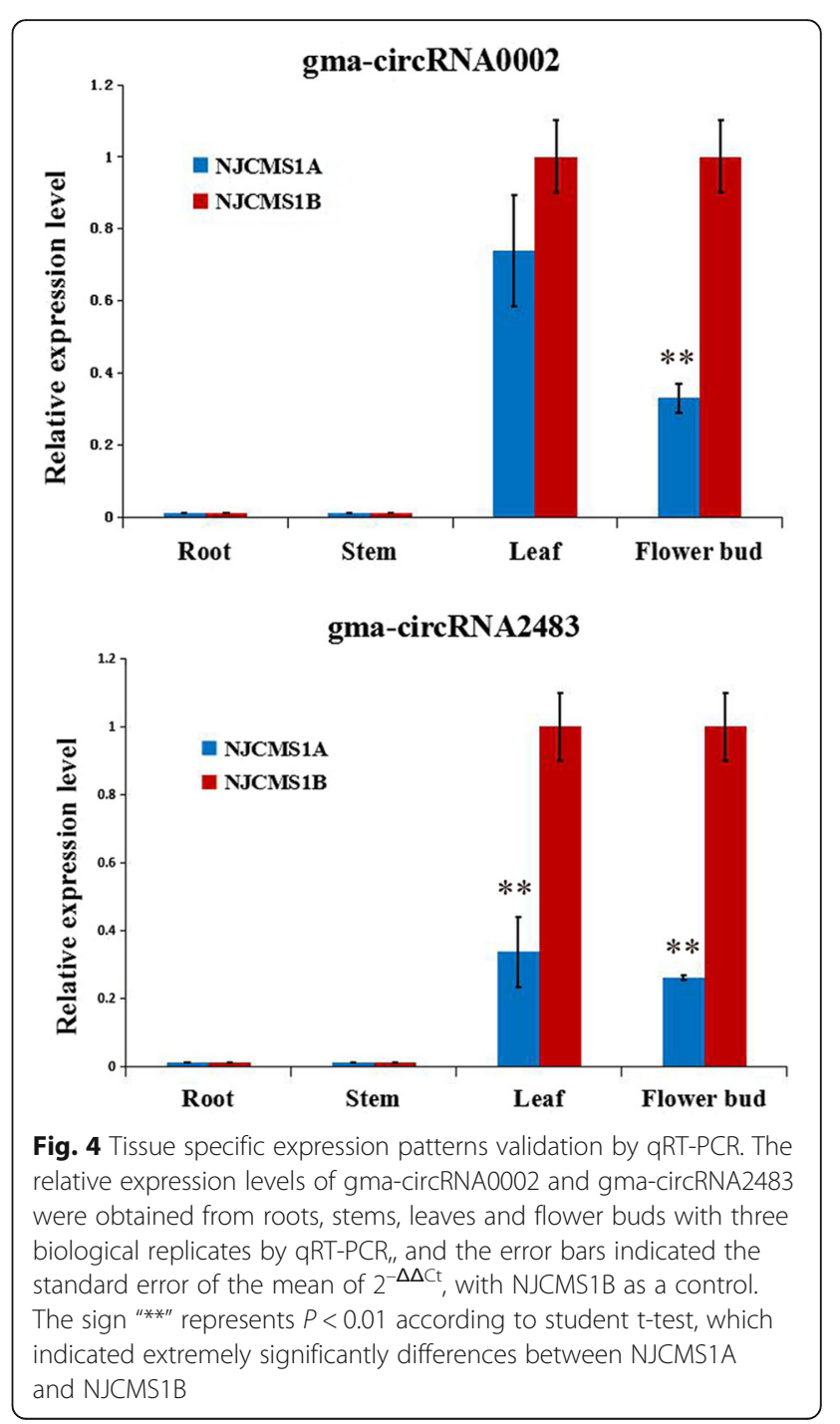


(GO:0065007), and cellular process (GO:0009987) (Fig. 5). Interestingly, a fraction of parental genes was classified into the categories of reproduction (GO:0000003) and reproductive process (GO:0022414). Among the cellular components, cell (GO:0005623), cell part (GO:0044464), and organelle (GO:0043226) accounted for a large proportion. In the molecular functions, the two main categories were binding (GO:0005488) and catalytic (GO:0003824). GO analysis of the parental genes showed that the differentially expressed circRNAs from NJCNS1A and NJCMS1B were associated with various functions in different biological processes, cellular components, and molecular function, indicating that circRNAs may play an important role in the fertility of soybean. KEGG pathway analysis identified 7 pathways including valine, leucine and isoleucine degradation, selenocompound metabolism, RNA transport, synthesis and degradation of ketone bodies, ascorbate and aldarate metabolism, ubiquitin mediated proteolysis, and porphyrin and chlorophyll metabolism (Additional file 7). These pathways are mainly related to amino acid degradation and material metabolism.

\section{Putative functions of parental genes of differentially expressed circRNAs in flower development and male fertility}

Based on the gene annotation of A. thaliana, the function of many parental genes were related to flower development and male fertility. We randomly enumerated 20 parental genes (Table 2) and selected four of them to describe their functions in detail. Glyma.06G173500, the parental gene of gma-circRNA0717, was a homolog of the Glucan Synthase-Like 8 (GSL8) gene in A. thaliana.
GSL8 and Glucan Synthase-Like 10 (GSL10) were two members of the A. thaliana GSL gene family, which are independently required for male gametophyte development and plant growth. Experiments showed that GSL8 and GSL1O T-DNA insertions led to pollen sterility [49]. GLS8 was believed to be involved in the synthesis of cell wall component callose [50]. It was hypothesized that gma-circRNA0717 may play a vital role in male gametophyte development. Glyma.13G230500, the parental gene of gma-circRNA1856, was an ortholog of the maternal effect embryo arrest 18 (MEE18) gene in A. thaliana. Mutation of MEE18 may affect pollen gametogenesis, pollen germination, pollen tube growth, polarity or guidance, and pollen tube-embryo sac interactions or fertilization [51]. $\mathrm{X}$-ray induced 1 gene (XRI1) was a novel DNA repair factor and was essential for male and female meiosis, and homozygous XRI1 mutants caused complete sterility in $A$. thaliana [52]. In this study, Glyma.18G164900, the parental gene of gma-circRNA2481, was a homolog of the XRI1 gene. Glyma.18G118100, the parental gene of gmacircRNA2454, was a homolog of squalene epoxidase 1 (SQE1) gene in A. thaliana. Previous studies showed that $S Q E 1$ mutants displayed severe growth defects in A. thali$a n a$, including short stature, short roots, and complete infertility [53]. We speculated that differences in circRNAs expression levels may influence the functions of pollen and male gametophytes, and result in CMS in NJCMS1A by interacting with their parental genes.

\section{Characterization of binding miRNAs of differentially expressed circRNAs}

CircRNAs have been reported to act as miRNA sponges regulating gene expression [25]. To verify if circRNAs

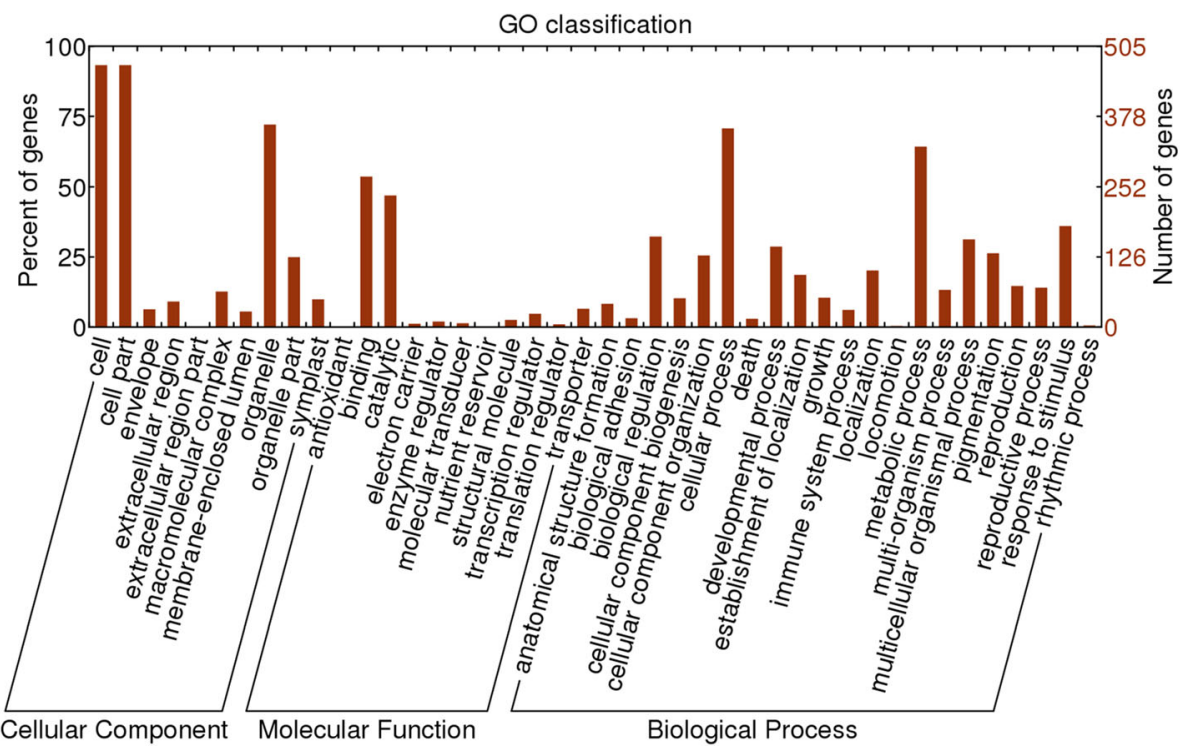

Fig. 5 Gene Ontology (GO) annotation of parental genes of the differentially expressed circRNAs between NJCMS1A and NJCMS1B 
Table 2 Twenty fertility-related parental genes of differentially expressed circRNAs

\begin{tabular}{|c|c|c|c|}
\hline circRNAs ID & Parental genes & $\begin{array}{l}\text { Homologue Genes } \\
\text { (Arabidopsis thaliana) }\end{array}$ & Function description \\
\hline gma-circRNA0457 & Glyma.04G155600 & AT2G24120 & male gametophyte development \\
\hline gma-circRNA0717 & Glyma.06G173500 & AT2G36850 & male gametophyte development; callose component \\
\hline gma-circRNA1324 & Glyma.09G266600 & AT3G61050 & male gametophyte development; pollen tube growth \\
\hline gma-circRNA2270 & Glyma.16G218700 & AT5G22110 & male gametophyte development; embryo development \\
\hline gma-circRNA2801 & Glyma.20G205100 & AT1G06750 & male gametophyte development; pollen tube growth \\
\hline gma-circRNA0057 & Glyma.01G140600 & AT1G71820 & pollen germination; pollen tube growth \\
\hline gma-circRNA0685 & Glyma.06G135600 & AT3G03810 & pollen tube development; chloroplast component \\
\hline gma-circRNA0914 & Glyma.07G002300 & AT1G78900 & pollen development \\
\hline gma-circRNA1244 & Glyma.09G103400 & AT2G37270 & pollen tube development \\
\hline gma-circRNA1301 & Glyma.09G194900 & AT5G65930 & pollen germination; plasma membrane component \\
\hline gma-circRNA1432 & Glyma.10G217200 & AT1G76850 & pollen germination; pollen tube growth \\
\hline gma-circRNA1856 & Glyma.13G230500 & AT2G34090 & pollen gametogenesis; pollen germination; pollen tube-embryo sac interactions \\
\hline gma-circRNA2481 & Glyma.18G164900 & AT5G48720 & pollen development; DNA repair; male meiosis \\
\hline gma-circRNA2791 & Glyma.20G182800 & AT3G10380 & pollen germination; pollen tube growth \\
\hline gma-circRNA1934 & Glyma.14G1 19800 & AT1G43850 & flower development; embryo development \\
\hline gma-circRNA2102 & Glyma.15G000300 & AT1G16710 & flower development; protein amino acid acetylation \\
\hline gma-circRNA2454 & Glyma.18G118100 & AT1G58440 & flower development; endomembrane system component \\
\hline gma-circRNA2637 & Glyma.19G212600 & AT4G02300 & flower development; cell wall component \\
\hline gma-circRNA0464 & Glyma.04G163400 & AT5G61150 & negative regulation of flower development \\
\hline gma-circRNA2163 & Glyma.15G265300 & AT1G79280 & negative regulation of flower development; stamen development \\
\hline
\end{tabular}

have a similar function in soybean, we predicted potential binding sites of miRNAs of the differentially expressed circRNAs. We observed that 72 differentially expressed circRNAs, of which 28 were up-regulated and 44 were down-regulated, contained 83 predicted circRNA-binding miRNAs. Of these circRNAs, only 24 had two to four miRNA binding sites (Additional file 8). Based on the predicted results, a circRNA-miRNA interaction network was delineated by Cytoscape (Fig. 6). The results showed that a single circRNA could target different miRNAs; for example, gma-circRNA0534 targeted to 16 miRNAs. Meanwhile, a single miRNA could be targeted by diverse circRNAs. For instance, gma-miR1533 was targeted by 27 circRNAs.

Among the predicted miRNAs, three miRNAs (gma-miR169e, gma-miR4349, and gma-miR4993) were differentially expressed in our previous study [11]. In addition, we found that some miRNAs, such as miR156, miR162, miR169, and miR172, were important for pollen development and male fertility in soybean. miR156 is one of the highly conserved miRNA families, which was first reported in A. thaliana [54]. Squamosa promoter binding like $(S P L)$ family proteins are involved in almost all physiological and biochemical processes such as morphogenesis, development stage transition, sporulation, flower and fruit development, external stress response, and hormone signal transduction [55]. Previous studies showed that miR156 regulated the development stage transition [56], flowering process [57], fertility maintenance [58], and fruit ripening [59] in plants by regulating members of the SPL family. Gma-circRNA0888, gmacircRNA1395, and gma-circRNA1746 could all target gma-miR156aa and gma-miR156z, which are members of miR156 family and probably involved in flower development. Furthermore, gma-circRNA1001 was predicted to target gma-miR162a, gma-miR162b, and gma-miR162c. NADP-dependent isocitrate dehydrogenase $(N A D P-I C D H)$ was targeted by gma-miR162a, gma-miR162b, and gma-miR162c according to the degradome analysis performed prior by NJCMS1A and NJCMS1B [11]. NADPH is a key cofactor in the cellular redox homeostasis, and is essential in the metabolism of reactive oxygen species (ROS) [60]. In $A$. thaliana, $N A D P-I C D H$ activity is regulated by molecules involved in ROS, including hydrogen peroxide $\left(\mathrm{H}_{2} \mathrm{O}_{2}\right)$ [61]. Our previous study showed that these three miRNAs had no significant differences in expression levels between the NJCMS1A and NJCMS1B, but the target gene NAD$P-I C D H$ was down-regulated in NJCMS1A as revealed by qRT-PCR analysis [11]. The decreased NADPH level 


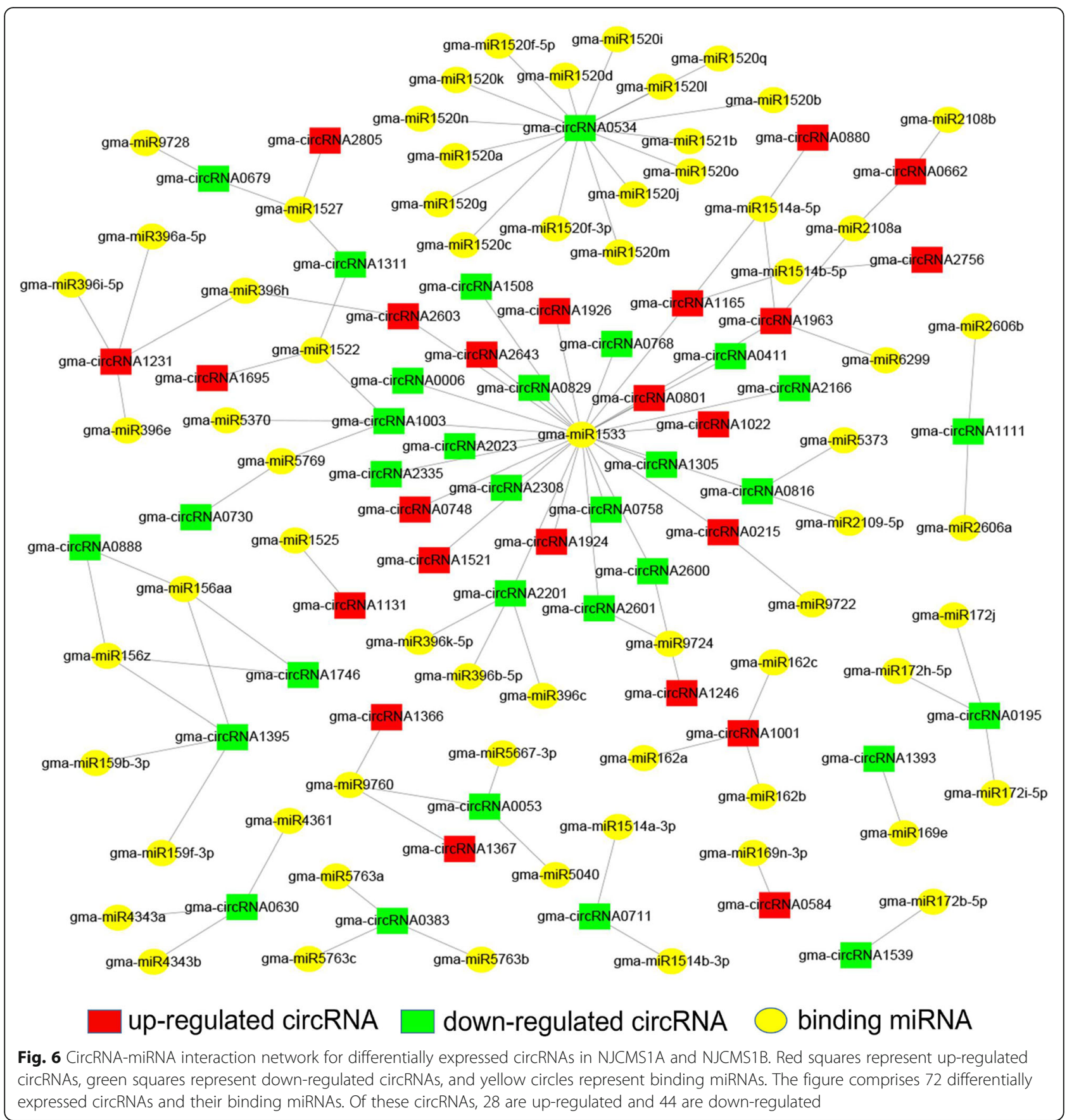

may result in significant ROS accumulation and male sterility in soybean. Moreover, gma-miR169e was targeted by gma-circRNA1393, and gma-miR169e could target dihydrolipoyl dehydrogenase (E3), an important part of the pyruvate dehydrogenase complex. Pyruvate dehydrogenase complex is a critical pathway that supports energy generation for pollen and pollen tube growth [62]. In our previous study, qRT-PCR analysis showed that the target gene E3 was down-regulated in NJCMS1A [11]. In addition,
gma-circRNA0195 was predicted to target gam-miR172j, which could target Glyma.01 g188400, the homolog of the APETALA2 (AP2) of A. thaliana. In A. thaliana, miR172 could control flowering time and floral organ formation by regulating expression of the AP2-like transcription factor. A previous study indicated that over-expression of miR172 caused flower development abnormalities, and its phenotype was similar to ap 2 mutants, leading to abnormal gametophyte development [63]. 
Functional annotation of predicted target mRNAs

To further explore the role of circRNAs in CMS in soybean, the 83 binding miRNAs of differentially expressed circRNAs were used to predict their possible target mRNAs by psRobot with the default parameters. In total, 1166 target mRNAs were predicted and further used for functional analysis (Additional file 8). A direct acyclic graph (DAG) of biological processes was obtained using the agriGO online server (Fig. 7). The DAG can indicate submitted terms and the inter-relationships between terms. From this figure, we could identify several pathways with significant enrichment, especially those involved in signal transduction and programmed cell death (PCD). A previous study showed that pollen development depended on the interaction of multiple signaling pathways, in which calmodulin was a key element [64]. Disruption of signaling pathways might cause abnormal pollen development, resulting in male sterility in soybean. In addition, PCD in plants is a cellular process similar to apoptosis, which contains fragmentation of nuclear DNA and is controlled by mitochondrion-driven signals [3]. Plant PCD plays a role in development processes such as senescence, seed germination, organ development, root tip elongation, xylem and aerenchyma formation, and disease resistance [65]. The development of plant male gametophytes in anthers requires cooperative interactions between sporophytic (anther wall) and gametophytic (microspore) cells as well as proper PCD-controlled cellular degeneration of the tapetum and the anther wall tissue [66]. Therefore,

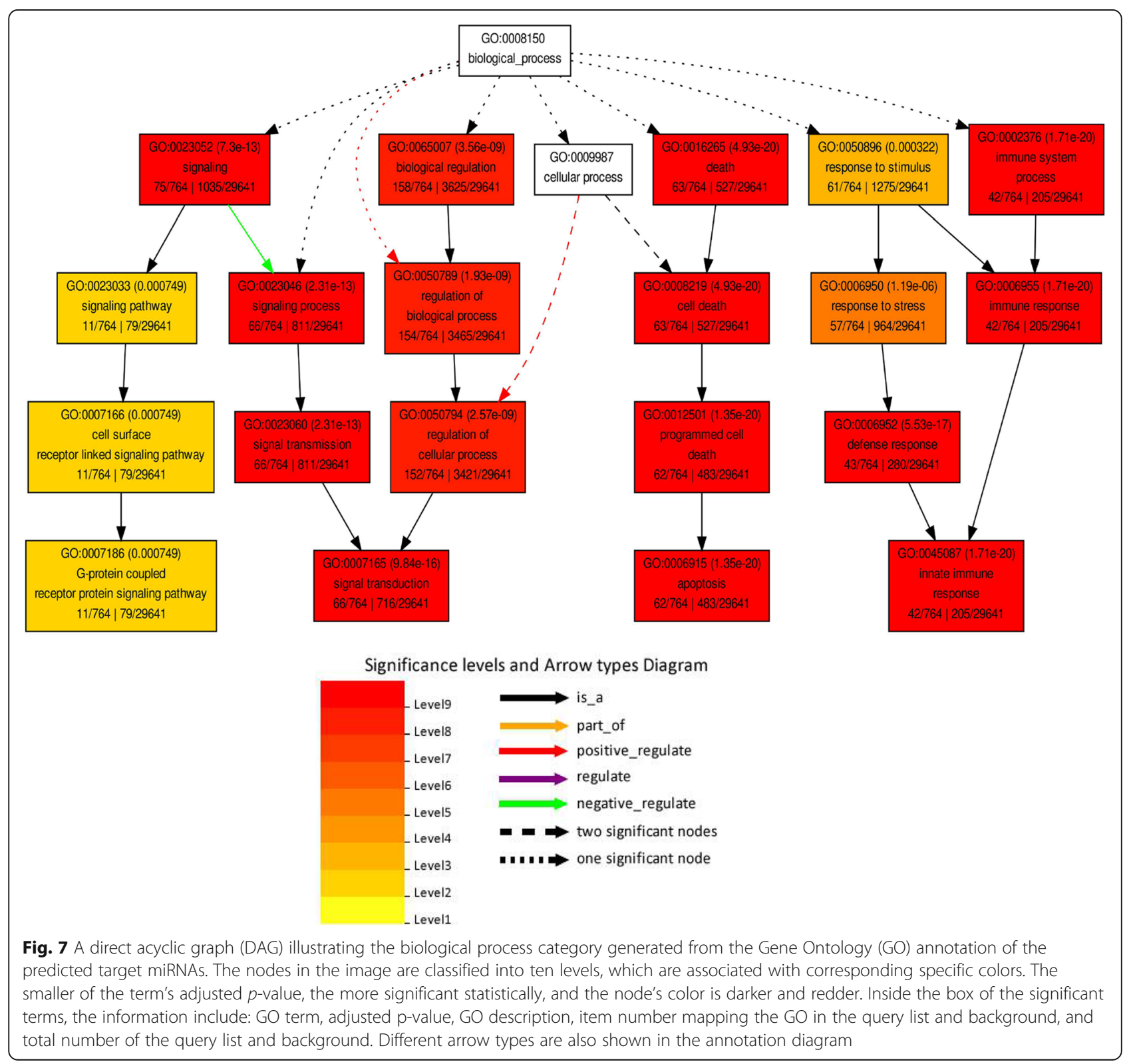


premature or delayed PCD leads to abnormality of pollen development and tapetal function, and even male sterility [67-69]. For example, the PET1-CMS cytoplasm in sunflower causes premature PCD of the tapetal cells, which then leads to abnormal anther development [70]. The KEGG pathway analysis was also used to further explore the function of predicted target mRNAs of differentially expressed circRNAs. The target mRNAs were significantly enriched in six pathways: beta-Alanine metabolism, fatty acid degradation, lysine degradation, ascorbate and aldarate metabolism, limonene and pinene degradation, and mRNA surveillance pathway (Table 3). Several studies have shown that in most plant species, the contents of amino acids, proteins, and soluble sugars in male sterile lines were lower than those in their maintainer lines [71, 72]. In this study, the pathways of amino acid degradation and material metabolism may be important causes of CMS in soybean. GO categories and KEGG pathway analyses showed that a large number of target mRNAs were involved in pollen development and male fertility, which implied that the differentially expressed circRNAs might play an important role in CMS of soybean.

\section{Prediction of protein-coding potential of soybean circRNAs}

Recent studies have demonstrated that some circRNAs can be translated into polypeptides or proteins by translation initiation element internal ribosome entry site (IRES) [27]. To verify whether soybean circRNAs have a similar function, we predicted the protein-coding potential by blasting sequences of the circRNAs to all the IRES sequences at the website. A total of 165 soybean circRNAs contained at least one IRES element and an open reading frame (Additional file 9), which might have protein-coding potential. Furthermore, conserved domains of the possible protein-coding products were predicted by Conserved Domain Database (Additional file 10) [73], which might have important functions. Recent studies have shown that the protein-coding products of circRNAs can influence the function of their parental genes by interacting directly or indirectly with them $[74,75]$.

Table 3 KEGG pathway enrichment of target mRNAs of differentially expressed circRNAs in soybean

\begin{tabular}{llll}
\hline Pathway ID & Description & Gene count & $P$-value \\
\hline gm×00410 & beta-Alanine metabolism & 9 & 0.004 \\
gm×00071 & Fatty acid degradation & 7 & 0.027 \\
gm×00310 & Lysine degradation & 5 & 0.030 \\
gm×00053 & Ascorbate and aldarate metabolism & 6 & 0.032 \\
gm×00903 & Limonene and pinene degradation & 3 & 0.034 \\
gmx03015 & mRNA surveillance pathway & 12 & 0.045 \\
\hline
\end{tabular}

Pathway with the threshold of $P$-value $<0.05$ was listed
In this study, some parental genes of circRNAs with protein-coding potential were associated with flower and pollen development. For example, gma-circRNA0736 was predicted to encode an 81- amino acid protein, and its parental gene was a homolog of VERNALIZATION INDEPENDENCE 4 (VIP4) in A. thaliana. The VIP4 mutants could cause slightly early flowering and variable fertility under standard growth conditions [76]. GmacircRNA1793 was predicted to encode a 154-amino acid protein, and its parental gene was a homolog of no pollen germination related 2 (NPGR2) in A. thaliana. The NPGR2 encodes a calmodulin-binding protein that is essential for pollen germination [77]. The protein-coding products of these circRNAs may affect flower and pollen development in soybean by affecting the function of the parental genes.

\section{Conclusion}

In this study, a total 2867 circRNAs, of which 1009 were differentially expressed between the soybean CMS line NJCMS1A and its maintainer NJCMS1B, were identified by high-throughput deep sequencing. Tissue specific expression patterns were verified by quantitative real-time PCR. The parental genes of differentially expressed circRNAs were mainly enriched in biological processes such as metabolic process, biological regulation, and reproductive process. A large number of parental genes were related to flower development and male fertility. A total of 83 binding miRNAs were predicted among the differentially expressed circRNAs, which included well-known flower and pollen development-related miRNAs. The target mRNAs predicted for the 83 binding miRNAs were significantly enriched in signal transduction and programmed cell death. A total of 165 soybean circRNAs contained at least one IRES element and an open reading frame (ORF), indicating their potential to encode polypeptides or proteins. Our study indicated that circRNAs might participate in the regulation of flower and pollen development, which could provide a new insight into the molecular mechanisms of CMS in soybean.

\section{Additional files}

Additional file 1: Table S1. Divergent primers for validation of 12 randomly-selected differentially expressed circRNAs and qRT-PCR. (XLSX $10 \mathrm{~kb}$ )

Additional file 2: Table S2. The circRNAs identified in NJCMS1A and NJCMS1B. (XLSX $254 \mathrm{~kb})$

Additional file 3: Table S3. The homologous circRNAs identified in this study vs. previous study of Zhao et al. (XLSX $3455 \mathrm{~kb}$ )

Additional file 4: Figure S1. Venn diagram shows the number of tissue preferentially expressed circRNAs in different tissues of soybean. (PDF $93 \mathrm{~kb}$ ) 
Additional file 5: Table S4-1. The up-expressed circRNAs identified in NJCMS1A and NJCMS1B; Table S4-2. The down-expressed circRNAs identified in NJCMS1A and NJCMS1B. (XLSX $106 \mathrm{~kb})$

Additional file 6: Figure S2. Junction sites were confirmed by Sanger sequencing. (PDF $2526 \mathrm{~kb}$ )

Additional file 7: Table S5. KEGG pathway enrichment of parental genes of differentially expression circRNAs in soybean. (XLSX $9 \mathrm{~kb}$ )

Additional file 8: Table S6. Predicted circRNA-miRNA-mRNA connection for differentially expressed circRNAs in NJCMS1A and NJCMS1B. (XLSX 48 kb)

Additional file 9: Table S7. CircRNAs with protein-coding potential. (XLSX $42 \mathrm{~kb}$ )

Additional file 10: Table S8. Conserved domains of the predicted protein-coding products. (XLSX $72 \mathrm{~kb})$

\section{Abbreviations}

AP2: APETALA2; circRNA: circular RNA; CMS: Cytoplasmic male sterility; DAG: Direct acyclic graph; E3: dihydrolipoyl dehydrogenase; GO: Gene Ontology; GSL10: Glucan Synthase-Like 10; GSL8: Glucan Synthase-Like 8; IRES: Internal ribosome entry site; KEGG: Kyoto Encyclopedia of Genes and Genomes; m6A: N6-methyladenosine; MEE18: Maternal effect embryo arrest 18; miRNA: microRNA; NADP-ICDH: NADP-dependent isocitrate dehydrogenase; NPGR2: No pollen germination related 2; PCD: Programmed cell death; qRT-PCR: Quantitative real time PCR; ROS: Reactive oxygen species; SPL: Squamosa promoter-binding protein-like; SQE1: Squalene epoxidase 1; VIP4: VERNALIZATION INDEPENDENCE 4; WEGO: Web Gene Ontology Annotation Plot; XRI1: X-ray induced 1

\section{Acknowledgements}

We would thank Novogene Bioinformatics Technology Co.Ltd. (Beijing, China) for conducting the whole-genome circRNA sequencing.

\section{Funding}

This work was financially supported by grants from the National Key R\&D Program of China (2016YFD0101500, 2016YFD0101504), the National Hightech R\&D Program of China (2011AA10A105), and the Program for Changjiang Scholars and Innovative Research Team in University (PCSIRT_17R55, PCSIRT13073).

\section{Availability of data and materials}

The sequencing data have been deposited in the Sequence Read Archive (SRA) at the National Center for Biotechnology Information (NCBI) under accession number SRP160000.

\section{Authors' contributions}

$J G, S Y$, and $L C$ conceived and designed the experiments. $L C$ performed the experiments and analyzed the data. $L C, X D, H Z, T H, Y L, T W, X L$, and $L J$ contributed reagents, materials, and analysis tools. LC and SY wrote the paper. JG, SY, QS, and LC revised the paper. All authors read and approved the final manuscript.

\section{Ethics approval and consent to participate}

The plant materials were collected from germplasms bank of National Center for Soybean Improvement. The collection and usage of samples followed the ethics of the People's Republic of China.

\section{Consent for publication}

Not applicable.

\section{Competing interests}

The authors declare that they have no competing interests.

\section{Publisher's Note}

Springer Nature remains neutral with regard to jurisdictional claims in published maps and institutional affiliations.

\section{Author details}

Soybean Research Institute, National Center for Soybean Improvement, Key Laboratory of Biology and Genetic Improvement of Soybean (General, Ministry of Agriculture), State Key Laboratory of Crop Genetics and Germplasm Enhancement, Jiangsu Collaborative Innovation Center for Modern Crop Production, Nanjing Agricultural University, Nanjing 210095, China. ${ }^{2}$ Soybean Genomics and Improvement Laboratory, Beltsville Agricultural Research Center, USDA-ARS, Beltsville, MD 20705, USA.

Received: 27 March 2018 Accepted: 3 September 2018

Published online: 12 September 2018

\section{References}

1. Havey MJ. The use of cytoplasmic male sterility for hybrid seed production. Springer Netherlands. 2004;2004:623-634.

2. Hanson MR. Plant mitochondrial mutations and male sterility. Annu Rev Genet. 1991;25:461-86.

3. Chen L, Liu YG. Male sterility and fertility restoration in crops. Annu Rev Plant Biol. 2014;65:579-606.

4. Hu J, Wang K, Huang W, Liu G, Gao Y, Wang J, et al. The rice pentatricopeptide repeat protein RF5 restores fertility in Hong-Lian cytoplasmic male-sterile lines via a complex with the glycine-rich protein GRP162. Plant Cell. 2012;24:109-22.

5. Fujji S, Yamada M, Fujita M, Itabashi E, Hamada K, Yano K, et al. Cytoplasmicnuclear genomic barriers in rice pollen development revealed by comparison of global gene expression profiles among five independent cytoplasmic male sterile lines. Plant Cell Physiol. 2010;51:610-20.

6. Davis WH. Route to hybrid soybean production. 1985; US Patent No, 4545146.

7. Sun H, Zhao LM, Huang M. Studies on cytoplasmic-nuclear male sterile soybean. Chin Sci Bull. 1994;39:175-6.

8. Gai JY, Cui ZL, Ji DF, Ren ZJ, Ding DR. A report on the nuclear cytoplasmic male sterility from a cross between two soybean cultivars. Soy Genet Newsl. 1995;22:55-8.

9. Li JJ, Han SH, Ding XL, He TT, Dai JY, Yang SP, et al. Comparative transcriptome analysis between the cytoplasmic male sterile line NJCMS1A and its maintainer NJCMS1B in soybean (Glycine max (L.) Merr.). PLoS One. 2015;10:e0126771.

10. Li JJ, Ding XL, Han SH, He TT, Zhang H, Yang LW, et al. Differential proteomics analysis to identify proteins and pathways associated with male sterility of soybean using iTRAQ-based strategy. J Proteomics. 2016;138:72-82.

11. Ding XL, Li JJ, Zhang H, He TT, Han SH, Li YW, et al. Identification of miRNAs and their targets by high-throughput sequencing and degradome analysis in cytoplasmic male-sterile line NJCMS1A and its maintainer NJCMS1B of soybean. BMC Genomics. 2016;17:24.

12. Li YW, Ding XL, Wang X, He TT, Zhang H, Yang LS, et al. Genome-wide comparative analysis of DNA methylation between soybean cytoplasmic male-sterile line NJCMS5A and its maintainer NJCMS5B. BMC Genomics. 2017;18:596

13. Jeck WR, Sorrentino JA, Wang K, Slevin MK, Burd CE, Liu J, et al. Circular RNAs are abundant, conserved, and associated with ALU repeats. RNA. 2013; 19:141-57.

14. Salzman J, Gawad C, Wang PL, Lacayo N, Brown PO. Circular RNAs are the predominant transcript isoform from hundreds of human genes in diverse cell types. PLoS One. 2012;7:e30733.

15. Memczak S, Jens M, Elefsinioti A, Torti F, Krueger J, Rybak A, et al. Circular RNAs are a large class of animal RNAs with regulatory potency. Nature. 2013;495:333-8

16. Ye CY, Chen L, Liu C, Zhu QH, Fan L. Widespread noncoding circular RNAs in plants. New Phytol. 2015;208:88-95.

17. Danan M, Schwartz S, Edelheit S, Sorek R. Transcriptome-wide discovery of circular RNAs in archaea. Nucleic Acids Res. 2012;40:3131-42.

18. Innocenti N, Nguyen HS, D'hérouël AF, Aurell E. An observation of circular RNAs in bacterial RNA-seq data. arXiv preprint. 2016;arXiv(1606):04576.

19. Cazalla D, Yario T, Steitz JA. Down-regulation of a host microRNA by a herpesvirus saimiri noncoding RNA. Science. 2010;328(5985):1563-6.

20. Suzuki $H$, Tsukahara T. A view of pre-mRNA splicing from RNase $R$ resistant RNAs. Int J Mol Sci. 2014;15:9331-42.

21. Salzman J, Chen RE, Olsen MN, Wang PL, Brown PO. Cell-type specific features of circular RNA expression. PLoS Genet. 2013;9:e1003777. 
22. Rybak-Wolf A, Stottmeister C, Glažar P, Jens M, Pino N, Giusti S, et al. Circular RNAs in the mammalian brain are highly abundant, conserved, and dynamically expressed. Mol Cell. 2015;58:870-85.

23. Li Z, Huang C, Bao C, Chen L, Lin M, Wang $X$, et al. Exon-intron circular RNAs regulate transcription in the nucleus. Nat Struct Mol Biol. 2015;22:256-64.

24. Darbani B, Noeparvar S, Borg S. Identification of circular RNAs from the parental genes involved in multiple aspects of cellular metabolism in barley. Front Plant Sci. 2016;7:776.

25. Hansen TB, Jensen TI, Clausen BH, Bramsen JB, Finsen B, Damgaard CK, et al. Natural RNA circles function as efficient microRNA sponges. Nature. 2013:495:384-8

26. Huang R, Zhang Y, Han B, Bai Y, Zhou R, Gan G, et al. Circular RNA HIPK2 regulates astrocyte activation via cooperation of autophagy and ER stress by targeting MIR124-2HG. Autophagy. 2017;13:1722-41.

27. Legnini I, Timoteo GD, Rossi F, Morlando M, Briganti F, Sthandier O, et al. Circ-ZNF609 is a circular RNA that can be translated and functions in myogenesis. Mol Cell. 2017;66:22-37.

28. Yun Y, Fan X, Mao M, Song X, Ping W, Yang Z, et al. Extensive translation of circular RNAs driven by N6-methyladenosine. Cell Res. 2017;27:626-41.

29. Zuo J, Wang Q, Zhu B, Luo Y, Gao L. Deciphering the roles of circRNAs on chilling injury in tomato. Biochem Biophys Res Commun. 2016;479:132-8.

30. Wang $Y$, Yang M, Wei S, Qin F, Zhao H, Suo B. Identification of circular RNAs and their targets in leaves of triticum aestivum $L$. under dehydration stress. Front Plant Sci. 2017;7:2024.

31. Liu T, Zhang L, Chen G, Shi T. Identifying and characterizing the circular RNAs during the lifespan of Arabidopsis leaves. Front Plant Sci. 2017;8:1278

32. Wang Z, Liu Y, Li D, Li L, Zhang Q, Wang S, et al. Identification of circular RNAs in kiwifruit and their species-specific response to bacterial canker pathogen invasion. Front Plant Sci. 2017;8:413.

33. Lu T, Cui L, Zhou Y, Zhu C, Fan D, Gong H, et al. Transcriptome-wide investigation of circular RNAs in rice. RNA. 2015;21:2076-87.

34. Tan JJ, Zhou ZJ, Niu YJ, Sun XY, Deng ZP. Identification and functional characterization of tomato circRNAs derived from genes involved in fruit pigment accumulation. Sci Rep. 2017;7:8594.

35. Zhao W, Cheng YH, Zhang C, You QB, Shen XJ, Guo W, et al. Genome-wide identification and characterization of circular RNAs by high throughput sequencing in soybean. Sci Rep. 2017;7:5636.

36. Ding DR, Gai JY, Cui ZL, Yang SP, Qiu JX. Development and verification of the cytoplasmic-nuclear male sterile soybean line NJCMS1A and its maintainer NJCMS1B. Chin Sci Bull. 1999;44:191-2.

37. Ding DR, Gai JY, Cui ZL, Qiu JX. Development of a cytoplasmic-nuclear male-sterile line of soybean. Euphytica. 2002;124:85-91.

38. Zhou L, Chen J, Li Z, Li X, Hu X, Huang Y, et al. Integrated profiling of microRNAs and mRNAs: microRNAs located on Xq27.3 associate with clear cell renal cell carcinoma. PLoS One. 2010;5:e15224

39. Love Ml, Huber W, Anders S. Moderated estimation of fold change and dispersion for RNA-seq data with DESeq2. Genome Biol. 2014;15:550.

40. Wu HJ, Ma YK, Chen T, Wang M, Wang XJ. PsRobot: a web-based plant small RNA meta-analysis toolbox. Nucleic Acids Res. 2012;40:W22-8.

41. Shannon P, Markiel A, Ozier O, Baliga NS, Wang JT, Ramage D, et al. Cytoscape: a software environment for integrated models of biomolecular interaction networks. Genome Res. 2003;13:2498-504.

42. Ye J, Fang L, Zheng H, Zhang Y, Chen J, Zhang Z, et al. WEGO: a web tool for plotting GO annotations. Nucleic Acids Res. 2006;34:W293-7.

43. Du Z, Zhou X, Ling Y, Zhang Z, Su Z. agriGO: a GO analysis toolkit for the agricultural community. Nucleic Acids Res. 2010;38:W64-7.

44. Tian T, Liu Y, Yan H, You Q, Yi X, Du Z, et al. agriGO v2.0: a GO analysis toolkit for the agricultural community, 2017 update. Nucleic Acids Res. 2017; https://doi.org/10.1093/nar/gkx382.

45. Mao X, Cai T, Olyarchuk JG, Wei L. Automated genome annotation and pathway identification using the KEGG Orthology (KO) as a controlled vocabulary. Bioinformatics. 2005;21:3787-93.

46. Annecatherine $P$, Herve P. Translational control of gene expression: role of iress and consequences for cell transformation and angiogenesis. Prog Nucleic Acid Res Mol Biol. 2002;72:367-413.

47. Altschul SF, Madden TL, Schäffer AA, Zhang J, Zhang Z, Miller W, et al. Gapped blast and psi-blast: a new generation of protein database search programs. Nucleic Acids Res. 1997;25:3389-402.

48. Chen L, Zhang P, Fan Y, Huang J, Lu Q, Li Q, et al. Transposons modulate transcriptomic and phenotypic variation via the formation of circular RNAs in maize. BioRxiv. 2017; https://doi.org/10.1101/100578.
49. Töller A, Brownfield L, Neu C, Twell D, Schulze-Lefert P. Dual function of Arabidopsis glucan synthase-like genes GSL8 and GSL10 in male gametophyte development and plant growth. Plant J. 2008;54:911-23.

50. Chen XY, Liu L, Lee E, Han X, Rim Y, Chu H, et al. The Arabidopsis callose synthase gene GSL8 is required for cytokinesis and cell patterning. Plant Physiol. 2009;150:105-13.

51. Boavida LC, Shuai B, Yu HJ, Pagnussat GC, Sundaresan V, Mccormick S. A collection of ds insertional mutants associated with defects in male gametophyte development and function in Arabidopsis thaliana. Genetics. 2009:181:1369-85.

52. Dean PJ, Siwiec T, Waterworth WM, Schlögelhofer P, Armstrong SJ, West CE. A novel ATM-dependent $X$-ray-inducible gene is essential for both plant meiosis and gametogenesis. Plant J. 2009;58:791-802.

53. Lalanne E, Michaelidis C, Moore JM, Gagliano W, Johnson A, Patel R, et al. Analysis of transposon insertion mutants highlights the diversity of mechanisms underlying male progamic development in Arabidopsis. Genetics. 2004;167:1975-86.

54. Rhoades MW, Reinhart BJ, Lim LP, Burge CB, Bartel B, Bartel DP. Prediction of plant microRNA targets. Cell. 2002;110:513-20.

55. Gou JY, Felippes FF, Liu CJ, Weigel D, Wang JW. Negative regulation of anthocyanin biosynthesis in Arabidopsis by a miR156-targeted SPL transcription factor. Plant Cell. 2011;23:1512-22.

56. $\mathrm{Yu} \mathrm{JH}$, Zhao $Y X$, Qin $Y T$, Xiao HL. Discovery of MicroRNAs associated with the $S$ type cytoplasmic male sterility in maize. J Integr Agr. 2013;12:229-38.

57. Wang JW, Czech B, Weigel D. miR156-regulated SPL transcription factors define an endogenous flowering pathway in Arabidopsis thaliana. Cell. 2009;138:738-49.

58. Xing S, Salinas M, Höhmann S, Berndtgen R, Huijser P. miR156-targeted and nontargeted SBP-box transcription factors act in concert to secure male fertility in Arabidopsis. Plant Cell. 2010;22:3935-50.

59. Usami T, Horiguchi G, Yano S, Tsukaya H. The more and smaller cells mutants of Arabidopsis thaliana identify novel roles for SQUAMOSA PROMOTER BINDING PROTEIN-LIKE genes in the control of heteroblasty. Development. 2009;136:955-64.

60. Leterrier M, Barroso JB, Valderrama R, Corpas FJ. NADP-dependent isocitrate dehydrogenase from Arabidopsis roots contributes in the mechanism of defence against the nitro-oxidative stress induced by salinity. ScientificWorldJournal. 2012;2012:694740.

61. Leterrier M, Barroso JB, Palma JM, Corpas FJ. Cytosolic NADP-isocitrate dehydrogenase in Arabidopsis leaves and roots. Biol Plantarum. 2012;56:1-6.

62. Selinski J, Scheibe R. Pollen tube growth: where does the energy come from? Plant Signal Behav. 2014;9:e977200.

63. Chen X. A microRNA as a translational repressor of APETALA2 in Arabidopsis flower development. Science. 2004;303:2022-5.

64. Rato C, Monteiro D, Hepler PK, Malhó R. Calmodulin activity and CAMP signalling modulate growth and apical secretion in pollen tubes. Plant J. 2004:38:887-97.

65. Reape TJ, Mccabe PF. Apoptotic-like regulation of programmed cell death in plants. Apoptosis. 2010;15:249-56.

66. Hong M. Molecular genetic analyses of microsporogenesis and microgametogenesis in flowering plants. Annu Rev Plant Biol. 2005;56:393-434.

67. Papini A, Mosti S, Brighigna L. Programmed-cell-death events during tapetum development of angiosperms. Protoplasma. 1999;207:213-21.

68. Kawanabe T, Ariizumi T, Kawai-Yamada M, Uchimiya H, Toriyama K. Abolition of the tapetum suicide program ruins microsporogenesis. Plant Cell Physiol. 2006;47:784-7.

69. Ji C, Li H, Chen L, Xie M, Wang F, Chen Y, et al. A novel rice bHLH transcription factor, DTD, acts coordinately with TDR in controlling tapetum function and pollen development. Mol Plant. 2013;6:1715-8.

70. Balk J, Leaver CJ. The PET1-CMS mitochondrial mutation in sunflower is associated with premature programmed cell death and cytochrome $c$ release. Plant Cell. 2001;13:1803-18.

71. Mazzafera P. Biochemical characterization of anthers from male-sterile coffee plants. Revista Brasileira De Genetica. 1991;2:413-23.

72. Ma ZH, Sun CQ, Wang JH, Pan YP. Study on biochemical characteristics of hot pepper (Capsicum annuum) cytoplasmic male sterile line with yellow-green seedling marker. Acta Agriculturae Jiangxi. 2012;2012:26-30.

73. Marchler-Bauer A, Bo Y, Han L, He J, Lanczycki CJ, Lu S, et al. CDD/SPARCLE: functional classification of proteins via subfamily domain architectures. Nucleic Acids Res. 2017;45:D200-3. 
74. Yang YB, Gao XY, Zhang ML, Yan S, Sun CJ, Xiao FZ, et al. Novel role of FBXW7 circular RNA in repressing glioma tumorigenesis. J Natl Cancer Inst. 2017;110(3):1.

75. Zhang ML, Huang N, Yang XS, Luo JY, Yan S, Xiao FZ, et al. A novel protein encoded by the circular form of the SHPRH gene suppresses glioma tumorigenesis. Oncogene. 2018;37:1805-14.

76. Zhang $\mathrm{H}$, Van Nocker $\mathrm{S}$. The VERNALIZATION INDEPENDENCE 4 gene encodes a novel regulator of FLOWERING LOCUS C. Plant J. 2002;31:663-73.

77. Golovkin M, Reddy AS. A calmodulin-binding protein from Arabidopsis has an essential role in pollen germination. Proc Natl Acad Sci U S A. 2003;100:10558-63.

Ready to submit your research? Choose BMC and benefit from:

- fast, convenient online submission

- thorough peer review by experienced researchers in your field

- rapid publication on acceptance

- support for research data, including large and complex data types

- gold Open Access which fosters wider collaboration and increased citations

- maximum visibility for your research: over $100 \mathrm{M}$ website views per year

At BMC, research is always in progress.

Learn more biomedcentral.com/submissions 